\title{
Bayesian Inference for Predicting an Inter-individual Variation of Thermal Sensation Votes
}

\author{
Jongyeon Lim ${ }^{1}$, Yasunori Akashi ${ }^{1}$, Naoki Yoshimoto ${ }^{2}$ \\ ${ }^{1}$ School of Engineering, The University of Tokyo, Tokyo, Japan \\ ${ }^{2} \mathrm{R} \& D$ Group, Hitachi, Ltd., Tokyo, Japan
}

\begin{abstract}
This paper proposes a probabilistic model that can provide the inter-individual variation of thermal sensation under a certain thermal condition. In contrast to the conventional model of predicting thermal sensation votes (TSVs) deterministically, our method in which an ordinal probit regression is adopted can capture stochastic characteristics for TSVs of a large group model. Model parameters were estimated using a hierarchical Bayesian inference technique. The results, based on a subset of the ASHRAE Global Thermal Comfort Database II, show that the proposed model provides accurate prediction of TSVs' variation. The PMV-PPD relationship was compared among the proposed model, field data, and Fanger's model. Unlike Fanger's model that has the lowest PPD in the neutral prescribed by the PMV, the highest satisfaction of field data was shifted toward slightly cool side and our prediction model captures this skewed trend.
\end{abstract}

\section{Introduction}

Comfortable thermal environment is defined as thermal conditions acceptable to $80 \%$ or more of the occupants within a space (ASHRAE, 2017). Although the concept of acceptability is hardly defined by the standard, it is generally agreed within research community that acceptability is synonymous with satisfaction (de Dear \& Brager, 2002). Furthermore, satisfaction is known to be associated with thermal sensations of slightly warm, neutral, and slightly cool. It corresponds to the definition of PPD (Predicted percentage dissatisfied), which is expressed with the percentage of occupants with thermal sensations of other than above-mentioned three categories. That is, thermal conditions of PPD $\leq 20 \%$ is arguably comfortable. Based on the relationship between the predicted mean vote (PMV) and PPD, this comfort condition is roughly corresponds to the range of $\mathrm{PMV}=[-1,1]$. However, this does not necessarily mean a space which meets this comfort condition guarantees more than $80 \%$ of occupants' satisfaction as several studies have reported discrepancies between the PPD and actual percentage dissatisfied (Humphreys \& Fergus Nicol, 2002; Schiller, 1990; van Hoof, 2008).

The PMV represents a mean thermal sensation of a large group of people under static thermal condition, and the PPD is determined based on PMV. Although there are occupants with different thermal sensation characteristics in a space, this conventional index cannot capture inherent stochastic variability of the human thermal sensation. Additionally, considering actual thermal environments have uncontrolled factors unlike a laboratory measurement, the implementation of the conventional index as a criterion for space control would not achieve high levels of occupant satisfaction.

In this study, we propose a probabilistic modeling approach for predicting the inter-individual variation of thermal sensation which allows us to quantify individual differences of a large group under a certain environment. We have developed a statistical model using ordinal probit regression that can consider both measured (thermal) and non-measured factors for thermal sensation. Model parameters are inferred using a hierarchical Bayesian approach. The proposed model is capable of providing a reliable prediction of thermal sensation variation of a large group, resulting in better agreement with observational percentage dissatisfied compared with the prediction by using PPD.

\section{Prediction model: ordinal probit regression}

This paper considers modeling techniques for the predicting the distribution of a discrete thermal sensation (the 7-point scale; cold, cool, slightly cool, neutral, slightly warm, warm, and hot) at given thermal conditions prescribed by a continuous PMV value.

Assume that a large group of subjects are asked to provide the thermal sensation votes (TSVs) for a given thermal condition within the 7-point scale. To develop a plausible prediction model for TSV responses, the following question must be answered: how does the subject decide a discrete ordinal response? It may be reasonable to presume that he/she has some internal scale of thermal condition that varies on a continuous metric scale, rather than having sense to quantify a given condition as the PMV value. We name this a latent scale, which is denoted by $y^{*}$. Because TSV is divided into 7 categories, his/her six thresholds $\theta_{k}(k=1, \ldots, 6)$ on the continuous coordinate of $y^{*}$ should be estimated. This allows us to match his/her thermal sensation under a given thermal condition on the 7-point TSV scale, as shown in Figure 2.

The next step is defining a latent scale and thresholds. In various previous studies, a simple linear regression has used for mapping from the PMV to $y^{*}$ :

$$
y^{*}=\alpha_{0}+\alpha_{1} \times \mathrm{PMV}
$$




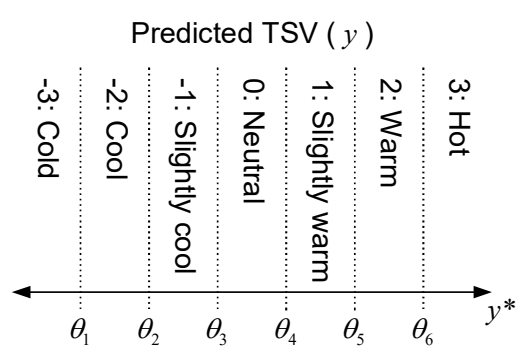

Figure 2: Mapping from a metric latent scale to the ordinal predicted TSV.

Simultaneously, an equal cognitive distance between thresholds is often assumed. Then, the predicted TSV $y$ is determined according to a pseudocode presented in Table 1.

Table 1 Pseudocode for conventionally predicting TSV.

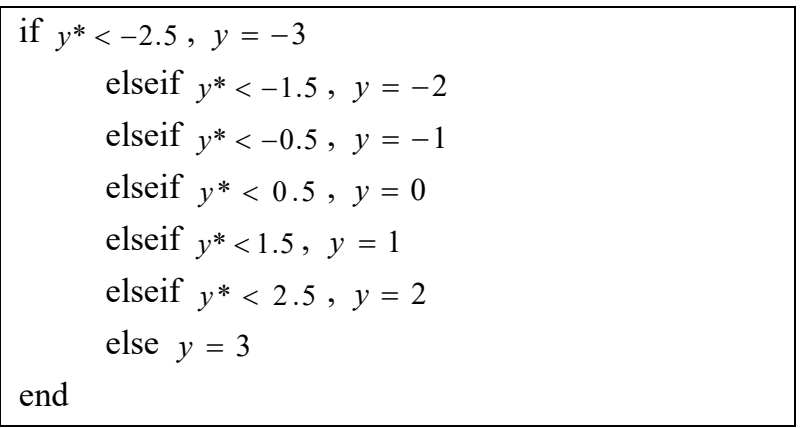

These are very reasonable approaches to predicting the thermal sensation which a hypothetical average person will feel, or the mean thermal sensation of a large group experiencing the same conditions. However, these may be insufficient to estimate a stochastic variation within the actual TSVs that is important for accurately evaluating the occupants' satisfaction.

Several studies (Höppe, 2002; Karyono, 2000) have reported that an inter-individual variation of TSVs within the field data is due to various non-measured (e.g., psychological and behavioral) factors. Despite these arguments, the TSVs' variation is often still ignored or simplified because of difficulties of being measured. This conventional approach yields the response probability for each TSV being deterministically established as 0 or 1 , based on the deterministically-defined $y^{*}$ (see Figure 1 (a)). In this paper, we approve the TSVs' variation and involve it in our prediction model. The proposed approach is schematically depicted as Figure 1 (b).
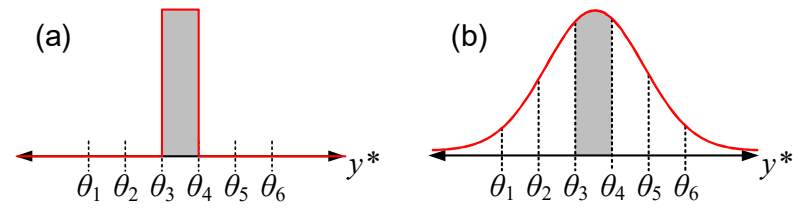

Figure 1: The difference of determining TSV between (a) the conventional method and (b) the proposed method. The response probability for each TSV corresponds to the area under the red line in thresholds; in other words, gray regions represents probability for TSV being neutral.
The $y^{*}$ is dispersed by various non-measured factors, and consequently the TSVs are probabilistically predicted by the response probability vector which consists of seven positive values that sum to 1 . We assume that the $y^{*}$ is normally distributed, then the response probability vector $\mathbf{Z}$ can be expressed as Eq. (2):

$$
\mathbf{Z}=\left[\begin{array}{c}
z_{1} \\
z_{2} \\
\vdots \\
z_{6} \\
z_{7}
\end{array}\right]=\left[\begin{array}{c}
\Phi\left(\theta_{1} \mid \mu, \sigma\right) \\
\Phi\left(\theta_{2} \mid \mu, \sigma\right)-\Phi\left(\theta_{1} \mid \mu, \sigma\right) \\
\vdots \\
\Phi\left(\theta_{6} \mid \mu, \sigma\right)-\Phi\left(\theta_{5} \mid \mu, \sigma\right) \\
1-\Phi\left(\theta_{6} \mid \mu, \sigma\right)
\end{array}\right]
$$

where vector element $z_{r}$ is the probability of TSV being $r$ $(r=1, \ldots, 7)$, $\Phi$ denotes the normal cumulative distribution function, and $\mu$ and $\sigma$ is a mean and a standard deviation of the normal distribution, respectively.

By defining the relationship of the PMV $-\mu$ and PMV $-\sigma$, we can yield the distribution of $y^{*}$ under a given thermal condition, which corresponds to mapping from the PMV to $y^{*}$. The $\mu$ is the expected value of $y^{*}$. It can describe the effect of thermal factors on TSVs because of sharing trends with PMV. At a large PMV, the value of $\mu$ would be large, consequently shifting the distribution of $y^{*}$ to the right. This means that the response probability for higher TSVs increases. We assumed $\mu$ to be expressed as a linear function of PMV:

$$
\mu=\beta_{0}+\beta_{1} \times \mathrm{PMV}
$$

The $\sigma$ is the variance of $y^{*}$. It can synthetically describe the effect of non-measured factors because of dispersing thermal sensation. Large values of $\sigma$ indicate that TSVs are more influenced by non-measured factors because they widen the distribution of $y^{*}$ and consequently lead to increasing the TSVs' uncertainty. The value of $\sigma$ is expected to be large in the middle of PMV's range and small at both ends for the following reasons: the distribution of TSVs is expected to be undispersed in extreme thermal conditions (extremely hot or extremely cold), depending on thermal factors; under conditions that are closer to thermal neutrality, the distribution of TSV will be scattered owing to the increased effect of various non-measured factors. This means that the homogeneous variance assumption for all thermal environments, which is assumed in a simple linear regression, is not realistic. We attribute this simplification to failure in predicting actual occupants' satisfaction. This $\sigma$ is a newly adopted model parameter to solve this limitation of the conventional method, and is assumed to be expressed with a Gaussian function:

$$
\sigma=\beta_{2} \times \exp \left(-\frac{\left(\mathrm{PMV}-\beta_{4}\right)^{2}}{2 \times \beta_{3}}\right)
$$

Our model represents the series of mapping process from PMV to ordinal response $y$. The distribution of $y^{*}$ that depends on the PMV is transformed into the $\mathbf{Z}$ using a thresholded cumulative normal function (see Eq. (2)). Then, we map this $\mathbf{Z}$ onto an ordinal variable $y$. This type 
of regression is called an ordinal probit regression (Kruschke, 2015).

In addition, for our ordinal probit regression model, the definition of six thresholds should be noted. As previously mentioned, an equal cognitive distance between thresholds is usually used (see Table 1). However, the relationships between adjacent thermal sensation are unknown. For the thresholds, we fix the two extreme thresholds as follows: $\theta_{1}=1.5$ and $\theta_{6}=6.5$, which imply that only the non-end thresholds are unknown and that estimates are according to two anchors.

In many previous studies (Oseland, 1998; and more), the predicted TSV was generally represented using a simple linear regression model. However, the conventional approach has some limitations as follows: (1) the homogeneous variance assumption for all thermal environments is not realistic; (2) the cognitive distances between adjacent thermal sensation responses are not the same and unknown (e.g., assuming TSV $=1$ as a reference point, the cognitive distance between 1 and 2, and 1 and 0 may not be the same,); (3) the actual TSVs are not deterministically established, but probabilistically determined. We attempted to overcome these limitations with the proposed regression model. Through positioning thresholds, $\theta_{2}, \ldots, \theta_{5}$, on a latent scale $y^{*}$ and estimating other model parameters, $\beta_{0}, \ldots, \beta_{4}$, appropriately, we can obtain a prediction of the TSVs' distribution of a large group in a given PMV condition.

\section{Bayesian inference using hierarchical model}

\section{Model structure}

The model parameter vectors $\boldsymbol{\beta}=\left(\beta_{0}, \ldots, \beta_{4}\right)$ and $\boldsymbol{\theta}=\left(\theta_{2}, \ldots\right.$, $\theta_{5}$ ) in previous section must be estimated appropriately for the prediction model to better describe the observation data $\mathbf{Y}=(x, y)$. We believe it is necessary to treat the TSV with a probabilistic approach and estimate the uncertainty of model parameters and its effect on the prediction of thermal sensation. For this purpose, we performed the analysis based on Bayesian statistics, within the framework of which we specified priors for the parameters and then converted prior uncertainty into posterior distributions by calculating the likelihood function in which the subject responses are reflected.

A primary application of Bayesian inference is in obtaining posterior distributions, which are expressed by the conditional probability distributions of parameters for a given dataset. Using Bayes' theorem, the posterior distribution of this problem is given by

$$
p(\boldsymbol{\beta}, \boldsymbol{\theta} \mid \mathbf{Y})=\frac{p(\mathbf{Y} \mid \boldsymbol{\beta}, \boldsymbol{\theta}) p(\boldsymbol{\beta}, \boldsymbol{\theta})}{p(\mathbf{Y})}
$$

where $p(\boldsymbol{\beta}, \boldsymbol{\theta} \mid \mathbf{Y})$ denotes the posterior distribution given $\mathbf{Y}$, $p(\mathbf{Y} \mid \boldsymbol{\beta}, \boldsymbol{\theta})$ denotes the likelihood of $\boldsymbol{\beta}$ and $\boldsymbol{\theta}$ given $\mathbf{Y}, p(\boldsymbol{\beta}, \boldsymbol{\theta})$ denotes the prior of model parameters, and $p(\mathbf{Y})$ denotes the evidence. Because $p(\mathbf{Y})$ does not depend on model parameters (assuming that it is constant), Eq. (5) can be rewritten as follows:

$$
p(\boldsymbol{\beta}, \boldsymbol{\theta} \mid \mathbf{Y}) \propto p(\mathbf{Y} \mid \boldsymbol{\beta}, \boldsymbol{\theta}) p(\boldsymbol{\beta}, \boldsymbol{\theta})
$$

Figure 3 schematically shows the dependencies across model parameters used in our model and statistical models.

\section{Likelihood and prior for the proposed model}

Based on Eq. (1), likelihood is given as follows:

$$
p(\mathbf{Y} \mid \boldsymbol{\beta}, \boldsymbol{\theta})=\prod_{n} z_{y_{n}}
$$

where $n$ is the number of observation data.

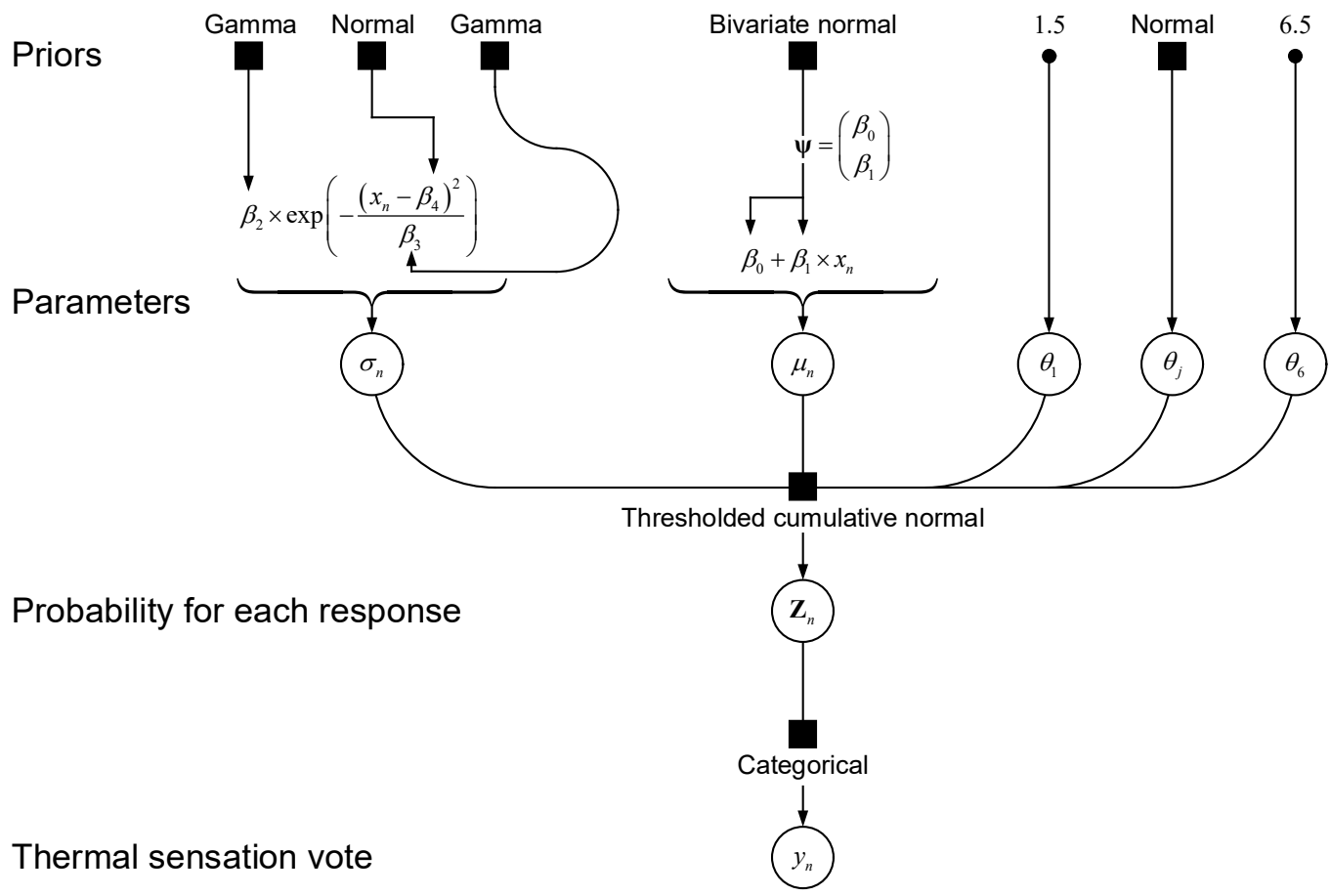

Figure 3: Model parameters structure. 
For priors, we selected non-informative priors such that they will have little impact on the inferences made relative to data. The prior distributions considered for different model parameters are as follows:

$$
\begin{aligned}
& \boldsymbol{\psi} \sim B V N\left(\left[\begin{array}{l}
0 \\
0
\end{array}\right],\left[\begin{array}{cc}
100 & 50 \\
50 & 100
\end{array}\right]\right) \\
& \beta_{2} \sim G(1.5,0.03) \\
& \beta_{3} \sim G(1.5,0.03) \\
& \beta_{4} \sim N(0,10) \\
& \theta_{j} \sim N(j+0.5,2) \quad(j=2,3,4,5)
\end{aligned}
$$

where $B V N(\mathbf{a}, \mathbf{C})$ denotes a bivariate normal prior with mean vector a and covariance matrix $\mathbf{C}, G(s, r)$ denotes a gamma prior with shape parameter $s$ and rate parameter $r$, $N(m, s d)$ denotes a normal prior with mean $m$ and standard deviation $s d$, and the symbol " " means "is distributed as."

\section{Estimation using the Markov chain Monte Carlo method}

The model parameters of interest are estimated using posterior distributions, which are determined by analytical integration. However, when there is more than one parameter of interest, we need to implement multiple integration, which is numerically very complex. This analytical approach makes evaluating the distribution even more difficult. Therefore, the Markov chain Monte Carlo (MCMC) method is a more appropriate approach for such parameter estimations. Gibbs sampling was used to evaluate the posterior distributions in this study, which were fully implemented using the ("JAGS - Just Another Gibbs Sampler," 2017). Following a burn-in of 40,000 iterations, the posterior distributions were monitored over an additional 600,000 iterations of the MCMC and were pooled over three parallel chains to ensure the validity of each sample sequence. To reduce the autocorrelation of sampled values and for the simplicity of post-processing, the samples obtained were thinned out at every 20th sample interval.

\section{Field survey data}

In order to estimate model parameters based on real world data, we used a subset of the ASHRAE Global Thermal Comfort Database II (Földváry Ličina et al., 2018), which was collected from air-conditioned office in Midland, UK $(n=9,205)$. This subset, which was also used in (Oseland, 1998), contains thermal sensation (the 7-point scale) and thermal acceptability ( 0 -unacceptable; 1 -acceptable) at given thermal conditions prescribed by PMV value.

For cross validation, we divided the data at a ratio of 8 (for training) to 2 (for validation). After examining various patterns of data division, we chose the model which seemed not to overfit for the training dataset. Figure 4 shows PMV-TSV relation obtained from training dataset $(n=7,364)$. The red line is a least-squares line, for which intercept and slope is 0.317 and 0.684 , respectively. The size of the circle in Figure 4 represents the number of data points of PMV-TSV pairs classified at $0.1 \mathrm{PMV}$ intervals. Because the data were collected in the conditioned office space, the data are concentrated around $0-0$ indicating the room condition was comfort for most people. On the other hand, at both ends of the PMV less than -1 and larger than 1 , very little data is available.

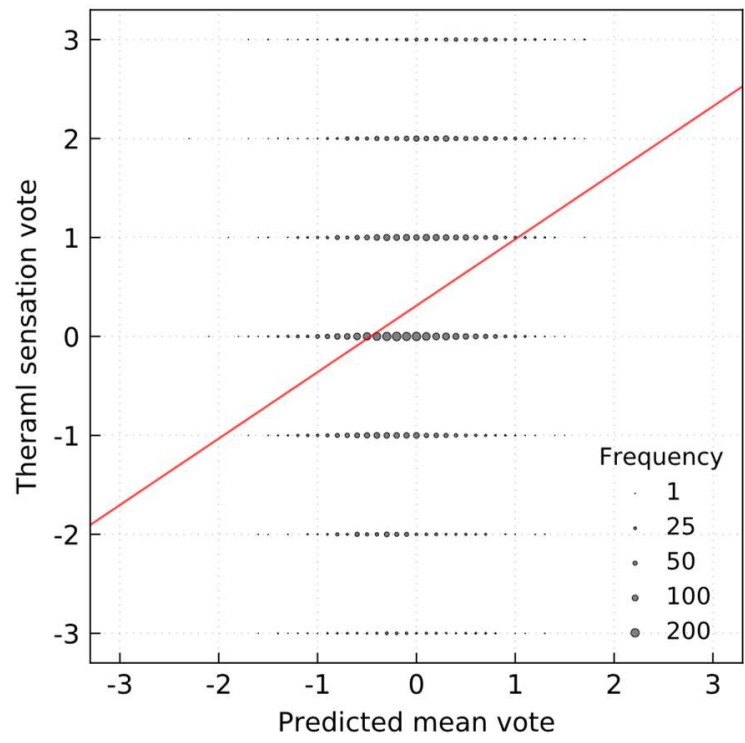

Figure 4: Distribution of field survey data used and linear regression for PMV-TSV relationship.

\section{Results}

The discussion in this section is about the model validation and comparison with the existing index. The detailed description for estimates of model parameters, such as the posterior distributions of model parameter vectors $\boldsymbol{\beta}$ and $\boldsymbol{\theta}$ obtained from the MCMC sampling datasets, is presented in Appendix.

\section{Thermal sensation profile}

Figure 5 shows the model-predicted probabilities for each response category of TSV under a given PMV, called thermal sensation profiles. Points estimated from the maximum a posteriori (MAP) for model parameters ( $\boldsymbol{\beta}$ and $\boldsymbol{\theta}$ ) were used for generating the profiles.

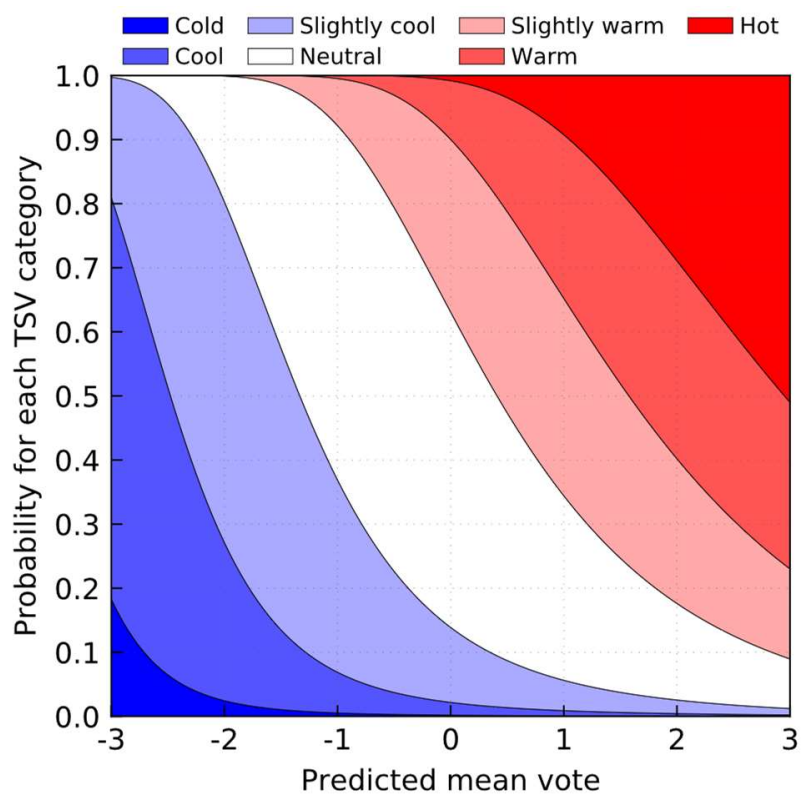

Figure 5: Thermal sensation profile generated from the proposed model. 
(a) Observation

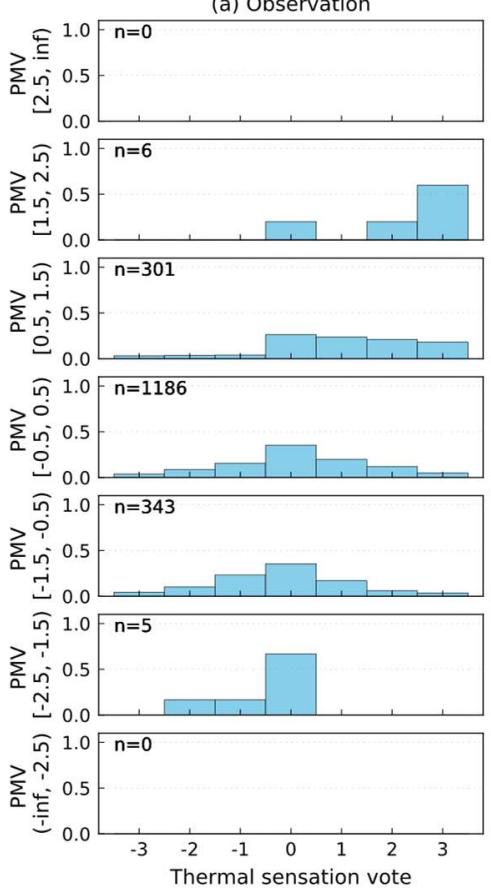

(b) Proposed model
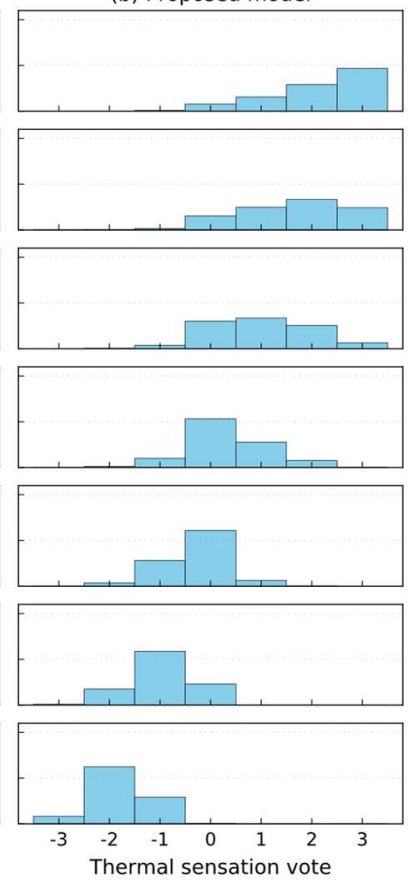

(c) Linear regression

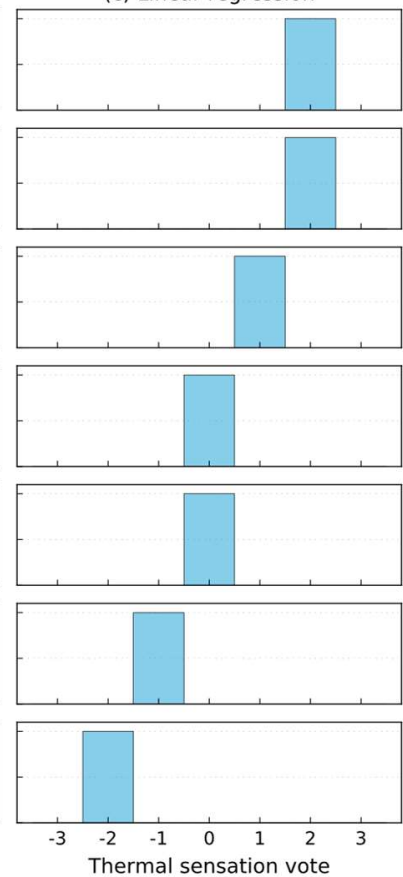

Figure 6: Response probability for each TSV category.

Our method allows the predicted TSVs under a given PMV to be probabilistically predicted by the response probability of each TSV category, compared with the simple linear regression (red line in Figure 4) yielding the deterministic estimate of the predicted TSV. This discrepancy in predictive ability is more evident from the following histograms.

Figure 6 shows the observational probabilities for each response category of TSV and the estimated probabilities obtained the simple linear regression and the proposed method. For both of training dataset and validation dataset, our method almost entirely reproduces the order relation of the response categories. This paper does not include the discussion on the predictions under thermal environment associated with the measured PMV being less than -2 or more than 2, since samples of the corresponding response categories are very few.

\section{Comparison with the existing index}

Figure 7 shows the relationship between PMV and modelpredicted percentage dissatisfied (MPD) with points estimated from the MAP and credible intervals (CI, 50\% and $95 \%$ ) of parameters, the relationship between PMV and percentage dissatisfied obtained from a field study data (OPD), and the existing PMV-PPD curve superimposed. For definitions of a dissatisfaction and an unacceptability, we follow a conventional approach as mentioned in previous section. That is, MPD and OPD mean the model-predicted percentage of occupants with thermal sensations of slightly warm, neutral, and slightly cool and the observational percentage of occupants who respond with an unacceptability, respectively. PPD does not reflect the PMV-OPD relation. On the other hand, the proposed model can capture the PMV-OPD relation, enclosing most of observational points in the prediction range for the $95 \% \mathrm{CI}$. This indicates that the variance of the thermal sensation votes due to non-measured factors and the resulting uncertainties of percentage dissatisfied are well-considered within the $95 \%$ CI estimated by the Bayesian inference.

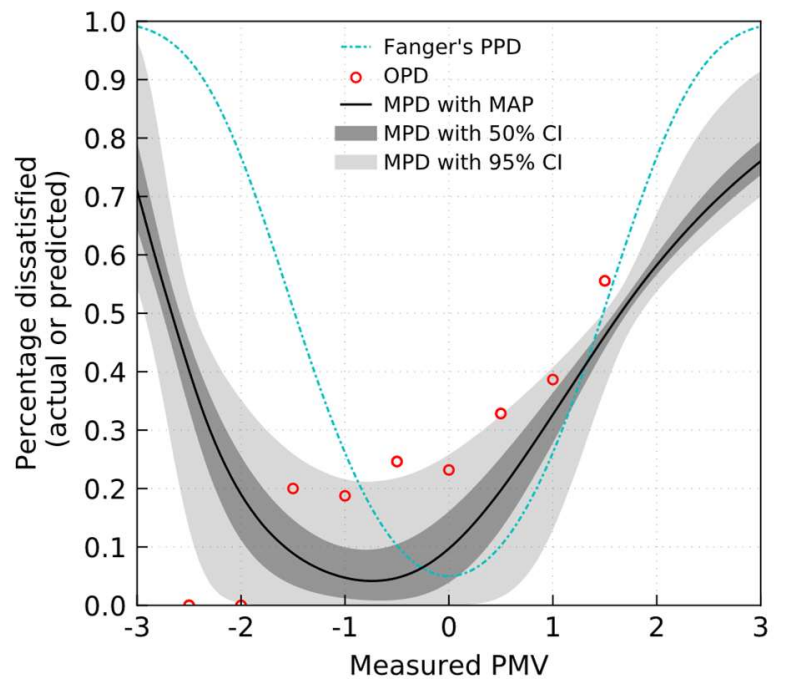

Figure 7: The relationship between the measured PMV and the occupants' satisfaction.

\section{Discussion}

In order to develop and apply optimal control strategies for thermal conditions acceptable to $80 \%$ or more of the occupants, a technique is needed to evaluate how much acceptability and/or satisfaction will be achieved under a given environment. The existing index, PMV-PPD relation, is based solely on the expected value of thermal sensation for an average person. However, there are 
occupants with various thermal sensation characteristics, which are affected by various non-measured factors, so that the thermal sensation will be dispersed. We argue that it is the limitation of the existing indices.

Our modeling approach takes the variance of thermal sensation due to non-measured factors into account. This allows to be capable of the prediction its distribution as well as its expected value (see Figure 6), resulting in predicting an unacceptability (expressed by the percentage dissatisfied) with improved accuracy. It can be helpful for future works to provide a more complete understanding of comfortable thermal environment and optimal control strategies.

In spite of our efforts to provide more precise estimates, the observational values are located on the upper limits for $95 \%$ CI. It possibly causes by an imprecise definition of a satisfaction and an acceptability that "it is associated with thermal sensations of slightly warm, neutral, and slightly cool." A threshold for a satisfaction may be between neutral and slightly warm/slightly cool. This hypothesis will push up the PMV-MPD curve (the black line in Figure 7) on the vertical axis, resulting in proving the proposed method to be remarkably accurate. This hypothesis testing requires the use of greater precision scales of thermal sensation, e.g., the 10-point scale. Of course, this approach requires further consideration. Dawes noted that the use of the detailed scale being more than the 5-point or 7-point cause no further improvement (Dawes, 2008). Additionally, the verbal expression of the 10-point scale becomes quite difficult.

\section{Conclusion}

Thermal satisfaction contributes to productivity in a daily life. Therefore, it is an important criterion for designers and engineers associated in building projects. Since Fanger defined the PMV and PPD as the index about thermal condition and occupants' satisfaction, they have been used worldwide to assess thermal environment. Additionally, a number of studies in both real world and climate chambers have been conducted in terms of its validity. Many studies supported his model while others, especially for real life situation, reported the discrepancies. We concluded that it is necessary, for accurately predicting occupants' satisfaction, to estimate the variance of thermal sensation as well as a mean of a large group. This study focused on the development and demonstration of a probabilistic modeling approach for predicting the inter-individual variation of thermal sensation. Unlike the existing simple linear regression method, we considered the effects of non-measured factors in addition to measured thermal factors on predicted thermal sensation. Model parameters were inferred using a hierarchical Bayesian approach. The proposed model is not only more promising than the simple linear regression method for explaining different thermal sensations under a same thermal condition, but it also provides information of reliable occupants' satisfaction.

\section{Acknowledgement}

This work was partly supported by Hitachi-UTokyo Laboratory (Hitachi-UTokyo Joint Research) "Habitat Innovation Project".

\section{Appendix}

Figure 8 and Figure 9 show the posterior probability density for $\boldsymbol{\beta}$ and $\boldsymbol{\theta}$ obtained from the MCMC sampling datasets, respectively. Although each parameter has a different distribution, all parameters estimated have unimodality and seem to be converged into the stationary distributions.
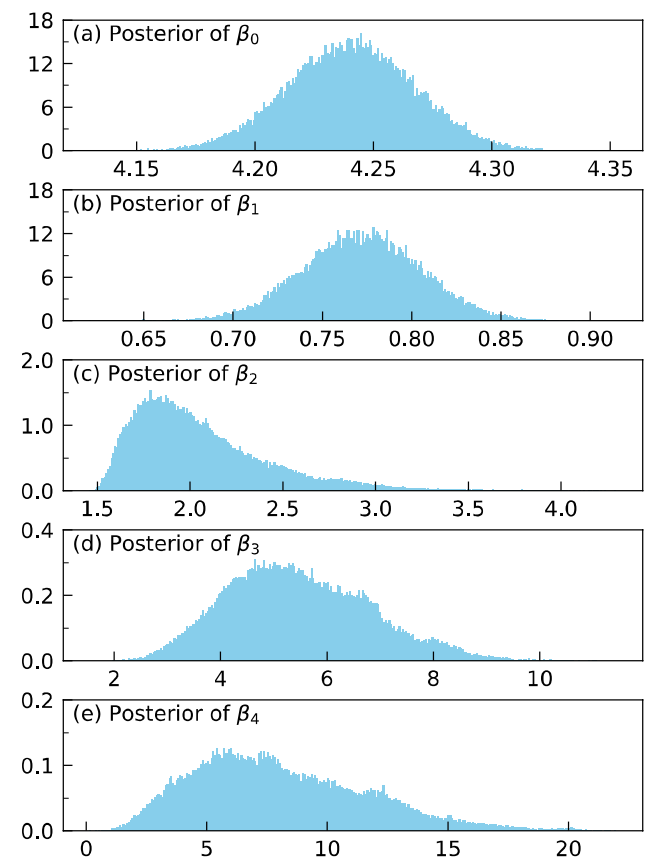

Figure 8: Posterior probability densities for each component of $\boldsymbol{\beta}$.

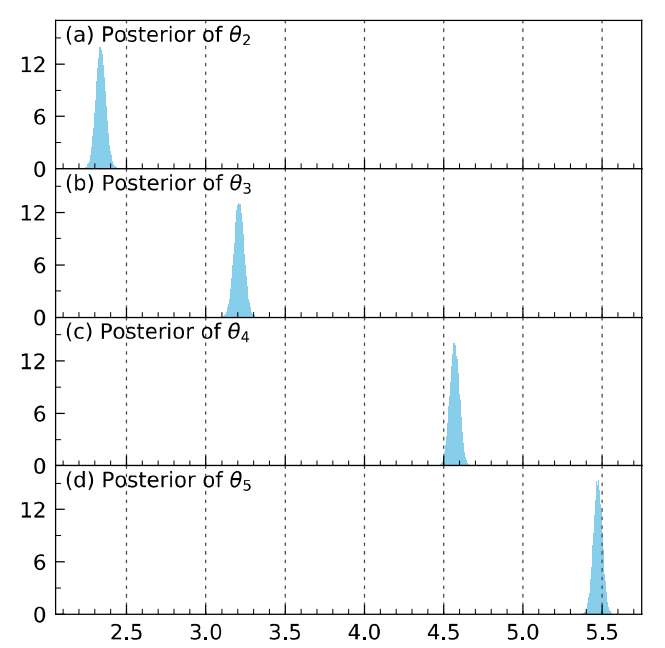

Figure 9: Posterior probability densities for each component of $\boldsymbol{\theta}$. 


\section{References}

ASHRAE. (2017). ASHRAE Standard 55 - Thermal environmental conditions for human occupancy.

Dawes, J. (2008). Do Data Characteristics Change According to the Number of Scale Points Used? An Experiment Using 5-Point, 7-Point and 10Point Scales. International Journal of Market Research, 50(1), 61-104.

de Dear, R. J., \& Brager, G. S. (2002). Thermal comfort in naturally ventilated buildings: revisions to ASHRAE Standard 55. Energy and Buildings, 34(6), 549-561.

Földváry Ličina, V., Cheung, T., Zhang, H., de Dear, R., Parkinson, T., Arens, E., ... Zhou, X. (2018). Development of the ASHRAE Global Thermal Comfort Database II. Building and Environment, $142,502-512$.

Höppe, P. (2002). Different aspects of assessing indoor and outdoor thermal comfort. Energy and Buildings, 34(6), 661-665.

Humphreys, M. A., \& Fergus Nicol, J. (2002). The validity of ISO-PMV for predicting comfort votes in every-day thermal environments. Energy and Buildings, 34(6), 667-684.
JAGS - Just Another Gibbs Sampler. (2017). Retrieved March 22, 2018, from http://mcmcjags.sourceforge.net

Karyono, T. H. (2000). Report on thermal comfort and building energy studies in Jakarta-Indonesia. Building and Environment, 35(1), 77-90.

Kruschke, J. K. (2015). Metric Predicted Variable with One Metric Predictor. In Doing Bayesian Data Analysis (2nd ed., pp. 477-508). Elsevier.

Lim, J., Akashi, Y., Song, D., Hwang, H., Kuwahara, Y., Yamamura, S., ... Itahashi, K. (2018). Hierarchical Bayesian modeling for predicting ordinal responses of personalized thermal sensation: Application to outdoor thermal sensation data. Building and Environment, 142, 414-426.

Oseland, N. A. (1998). Acceptable temperature ranges in naturally ventilated and air-conditioned offices. ASHRAE Transactions, 104(1), 1018-1030.

Schiller, G. E. (1990). A comparison of measured and predicted comfort in office buildings. ASHRAE Transactions, 96(1), 609-622.

van Hoof, J. (2008). Forty years of Fanger's model of thermal comfort: comfort for all? Indoor Air, 18(3), 182-201. 